\title{
The characteristics and novel clinical implications of CD4+CXCR5+Foxp3+ follicular regulatory T cells in breast cancer
}

\author{
Xianyuan Miao" ${ }^{1 \#}$, Qiusheng Guo ${ }^{1 \#}$, Zhiwen Pan $^{2,3}$, Xiaohong $\mathrm{Xu}^{2,3}$, Xiying Shao ${ }^{3,4}$, Xiaojia Wang \\ ${ }^{1}$ The Second Clinical Medical College of Zhejiang Chinese Medical University, Hangzhou, China; ${ }^{2}$ Department of Clinical Laboratory, Cancer \\ Hospital of the University of Chinese Academy of Sciences/Zhejiang Cancer Hospital, Hangzhou, China; ${ }^{3}$ Institute of Cancer and Basic Medicine \\ (ICBM), Chinese Academy of Sciences, Hangzhou, China; ${ }^{4}$ Department of Medical Oncology (Breast Cancer), Cancer Hospital of the University of \\ Chinese Academy of Sciences/Zhejiang Cancer Hospital, Hangzhou, China \\ Contributions: (I) Conception and design: X Shao, X Wang; (II) Administrative support: X Shao, X Wang; (III) Provision of study materials or \\ patients: Z Pan, X Xu; (IV) Collection and assembly of data: X Miao, Q Guo; (V) Data analysis and interpretation: X Miao, Q Guo; (VI) Manuscript \\ writing: All authors; (VII) Final approval of manuscript: All authors. \\ "These authors contributed equally to this work. \\ Correspondence to: Xiying Shao; Xiaojia Wang. Department of Medical Oncology (Breast Cancer), Cancer Hospital of the University of Chinese \\ Academy of Sciences/Zhejiang Cancer Hospital, Hangzhou, China. Email: shaoxy@zjcc.org.cn; wxiaojia0803@163.com.
}

Background: Follicular regulatory $\mathrm{T}$ cells (Tfr) are a subset of regulatory $\mathrm{T}$ cells (Tregs) that suppress the humoral immune response in the germinal center. They are associated with increased rates of disease stabilization and decreased autoantibody levels in a variety of tumor and autoimmune diseases. The binding of T-cell immunoglobulin mucin 3 (TIM-3) and its ligand on the surface of Tfr cells could result in the depletion of $\mathrm{T}$ lymphocytes and the termination of the immune response mediated by helper $\mathrm{T}$ cell 1 . However, the role of Tfr cells in breast cancer (BC) remains unclear.

Methods: In this study, we detected the expression of CD4+CXCR5+Foxp3+Tfr cells in the peripheral blood of 35 BC patients and 30 healthy control patients by flow cytometry, and analyzed the relationship between Tfr cells and the clinical characteristics of patients. In addition, the expression of TIM-3 on the surface of Tfr cells in 6 triple-negative BC (TNBC) patients was further investigated using mass spectrometry.

Results: We found a significant increase in Tfr cells in BC patients compared to healthy control patients $(23.47 \% \pm 9.70 \%$ vs. $10.99 \% \pm 4.68 \% ; \mathrm{P}=0.001)$. Notably, the increase was more significant in early stage than advanced stage TNBC patients $(28.52 \% \pm 10.75 \%$ vs. $18.69 \% \pm 5.19 \% ; \mathrm{P}=0.006)$, and there was a negative correlation between Tfr cells and serum lactate dehydrogenase (LDH) in early stage TNBC patients $(\mathrm{r}=-0.585 ; \mathrm{P}=0.008)$. Additionally, we found that the expression of Tfr cells was higher in TNBC patients than luminal BC patients $(28.25 \% \pm 10.11 \%$ vs. $18.5 \% \pm 8.15 \%$; $\mathrm{P}=0.028)$; however, there was no significant difference in expression in hormone receptor positive (HR+) BC and hormone receptor negative (HR-) BC $(\mathrm{P}=0.141)$ patients. Notably, the surface of Tfr cells of TNBC patients had higher levels of TIM-3 expression than those of healthy control patients $(3.93 \pm 0.92$ vs. $2.65 \pm 0.15$, respectively; $t=-3.02 ; \mathrm{P}<0.05)$, which the mass spectrometry showed were positively correlated with the intracellular Foxp 3 expression of $\mathrm{Tfr}$ cells $(\mathrm{r}=0.82$; $\mathrm{P}=0.036$ ).

Conclusions: Our results suggest that circulating Tfr cells and the expression of TIM-3 were significantly increased in $\mathrm{BC}$ patients, which were related to stage and histological type, and may be involved in the pathogenesis of $\mathrm{BC}$.

Keywords: Breast cancer (BC); follicular regulatory T cell (Tfr); T-cell immunoglobulin mucin 3 (TIM-3)

Submitted Jun 29, 2021. Accepted for publication Aug 16, 2021.

doi: $10.21037 / \mathrm{atm}-21-3848$

View this article at: https://dx.doi.org/10.21037/atm-21-3848 


\section{Introduction}

Follicular regulatory $\mathrm{T}$ cells (Tfr) cells are a recently identified class of regulatory $\mathrm{T}$ cells (Tregs) that maintain stable expression of ICOS, CXCR5, CD4 and Foxp3 (1). The main function of Tfr cells is to regulate humoral immunity in the germinal center by suppressing follicular helper T cells (Tfh) cells and B cells $(2,3)$. A lack of Tfr cells might result in greater germinal center reactions $(2,3)$. It has been confirmed that Tfr cells play a critical role in the development of non-small cell lung cancer (NSCLC), lymphoma, and other autoimmune diseases (4-6). Research has shown that Tfr cells are abundant in the lymph nodes of early stage diffuse large B cell lymphoma (DLBCL) patients, but reduced in advanced stage DLBCL patients; thus, Tfr cells may be strongly related to prognosis (7). It should be noted that T-cell immunoglobulin mucin 3 (TIM-3), one of the important immune suppressive receptors, could also co-express on the surface of Tfr cells with programmed death-1 (PD-1) (8). In the tumor microenvironment (TME), an obviously increased expression of TIM-3 could interact with $T$ cells by containing the ligand, and be involved in the development of tumors $(9,10)$.

Breast cancer (BC) is one of the most common malignant tumors in women, and approximately $25 \%$ of early-stage BC patients relapse within 5 years even with the implementation of surgery, chemotherapy, endocrine therapy, and other effective strategies, such as PD-1 immunotherapy $(11,12)$. The phase III IMpassion 130 trial (NCT02425891), which compared chemotherapy plus atezolizumab and chemotherapy, examined immunotherapy in the treatment of BC, and the Food and Drug Administration (FDA) has accelerated the approval of atezolizumab (13). Recently, more and more research has confirmed the inseparable relationship between the progression of $\mathrm{BC}$ and the immune system (14-16). Interestingly, the imbalance of $\mathrm{Tfr} / \mathrm{Tfh}$ cells has been reported to be the potential cause of BC (17). It is thought that Tfr cells might synergistically promote the formation of interleukin-10 (IL-10)-produced B cells in breast carcinoma (17). Additionally, recent studies have also shown that the co-blocking of PD-1 could further upregulate the expression of TIM-3 on the surface of Treg in TNBC, and TIM-3 may be a targeted biomarker related to the resistance of PD-1 blockers (18). Tfr cells are likely to mediate the immune escape of BC through the TIM-3 pathway. Thus, the identification of key molecular targets of Tfr cells could provide a new basis for the optimization of immunotherapy.
Table 1 Clinical characteristics of breast cancer (BC) patients

\begin{tabular}{lc}
\hline Characteristics & Number (\%) \\
\hline Age & $12(34.29)$ \\
$<40$ years & $12(34.29)$ \\
$40-60$ years & $11(31.43)$ \\
$>60$ years & \\
TNM stage & $17(48.57)$ \\
I-III & $18(51.42)$ \\
IV & \\
Histological cell type & $19(54.29)$ \\
HR- & $16(45.71)$ \\
HR+ & $12(34.29)$ \\
Triple-negative BC (TNBC) & $12(34.29)$ \\
Luminal & $7(20.00)$ \\
HER-2+ & \\
\hline
\end{tabular}

In this study, we sought to clarify the characteristics of circulating Tfr (cTfr) cells and TIM-3 in BC patients by measuring the frequency of Tfr cells. We also examined the expression of immune cells in TNBC to gain insights into the function of cTfr cells in BC. We present the following article in accordance with the MDAR reporting checklist (available at https://dx.doi.org/10.21037/atm-21-3848).

\section{Methods}

\section{Patients}

Thirty-five female BC patients and 30 age- and sexmatched healthy control patients were identified at the Cancer Hospital of the University of Chinese Academy of Sciences/Zhejiang Cancer Hospital from March 2020 to September 2020. The patients were confirmed to have invasive breast carcinoma by surgical resection or puncture, and the pathological stage was classified according to the international tumor $(\mathrm{T})$, nodes $(\mathrm{N})$, and metastases (M) staging system for BC. Seventeen patients had early $\mathrm{BC}$ and 18 patients had advanced $\mathrm{BC}$. In relation to the histological subtypes, 12 patients had TNBC, 12 patients had human epidermal growth factor receptor 2 positive (HER2+) BC, and 7 patients had luminal BC. The clinical characteristics of the BC patients are set out in Table 1. The lactate dehydrogenase $(\mathrm{LDH})$ records of these patients were 
Table 2 The expression of Tfr cells, B cells, and CD8+ T cells in the peripheral blood of triple-negative breast cancer (TNBC) and healthy control patients

\begin{tabular}{|c|c|c|c|c|c|c|c|}
\hline Group & $\mathrm{n}$ & \multicolumn{2}{|c|}{ Tfr cells } & \multicolumn{2}{|c|}{ B cells } & \multicolumn{2}{|c|}{ CD8+ $T$ cells } \\
\hline Control & 3 & $0.48 \pm 0.19$ & $0.15 \pm 0.05$ & $0.27 \pm 0.11$ & $0.78 \pm 1.0$ & $0.17 \pm 0.16$ & $5.48 \pm 2.12$ \\
\hline TNBC & 6 & $0.96 \pm 0.40$ & $0.20 \pm 0.09$ & $0.12 \pm 0.10$ & $0.28 \pm 0.17$ & $0.05 \pm 0.03$ & $4.42 \pm 1.94$ \\
\hline
\end{tabular}

retrospectively collected.

Additionally, 6 female TNBC and 3 age- and sexmatched healthy control patients were identified at our hospital from October 2020 to December 2020. Of these 6 patients, 3 were preoperative patients and 3 were recurrent and progressive patients.

All patients and healthy control patients were aged from 40 to 60 years old. Patients who had already received glucocorticoids, immunotherapy, or who had been diagnosed with other autoimmune diseases were excluded. $4 \mathrm{~mL}$-peripheral blood samples of patients were used in this research. All procedures performed in this study involving human participants were in accordance with the Declaration of Helsinki (as revised in 2013). The study was approved by institutional ethics board of Cancer Hospital of the University of Chinese Academy of Sciences/Zhejiang Cancer Hospital (No.: IRB-2020-14). Individual consent for this retrospective analysis was waived.

\section{Flow cytometry}

Peripheral blood mononuclear cells (PBMCs) were isolated from the $4 \mathrm{~mL}$-peripheral blood samples using Ficoll separation. The PBMCs were adjusted to $1 \times 10^{9} / \mathrm{L}$ in Roswell Park Memorial Institute Medium (RPMI) 1640 (containing 10\% fetal bovine serum) and incubated with fluorophore tagged anti-human CD3, CD4, and CXCR5 for $30 \mathrm{~min}$ at room temperature. The cells were then washed twice with phosphate buffered saline (PBS), and intracellular Foxp3 staining was performed with the Transcription Factor Staining Buffer Kit for $50 \mathrm{~min}$ at $4{ }^{\circ} \mathrm{C}$. After fixation and permeability, anti-Foxp 3 monoclonal antibody and fixed cells were incubated at $4{ }^{\circ} \mathrm{C}$ in the dark for $30 \mathrm{~min}$. Finally, the sample were washed again and analyzed at FACS Canto II.

To determine the expression of TIM-3 on the surface of Tfr cells in TNBC, the cells were stained with anti-CD4, CXCR5, CD25, CD19, CD8, and TIM-3 for 30 mins at room temperature (see Table 2). The other steps were the same as those outlined above. Finally, samples were analyzed on Helios Cytof System (Fluidigm, USA). All of the antibodies used were purchased from BD Biosciences (San Diego, CA, USA).

\section{Statistical analysis}

The data are expressed as mean \pm standard deviation (SD) and Boxplot. R Studio (version 3.6.2) was used for graphics production. The differences in the peripheral blood $\mathrm{Tfr}$ cells among different groups, BC stages, molecular types, and the expression of TIM-3 in TNBC were evaluated using $T$-tests. A $\mathrm{P}<0.05$ on both sides was considered statistically significant. Flowjo was used to draw the t-distributed stochastic neighbor embedding ( $t-S N E)$ diagram to show the expression distribution and content of surface antigens in the human peripheral blood immune cells.

\section{Results}

\section{Increased levels of Tfr cells in the peripheral blood of $B C$ patients}

To study the potential role of Tfr cells in BC, we quantitatively analyzed the differences of CD4+CXCR5+CD25+Foxp3+Tfr cells in the peripheral blood of 35 patients and 30 healthy age- and sex-matched control patients by flow cytometry (see Figure 1A). The patients' demographic and clinicopathologic characteristics are listed in Table 1. As Figure $1 B$ shows, the percentage of circulating CD4+CXCR5+Foxp3+Tfr cells was significantly higher in $\mathrm{BC}$ patients than healthy control patients $(23.47 \% \pm 9.70 \%$ vs. $10.99 \% \pm 4.68 \% ; \mathrm{t}=-6.59$; $\mathrm{P}=0.001)$. We also analyzed the percentage of cTfr cells in the peripheral blood of patients with different stages of BC. Our results showed that early-stage disease BC patients had a higher percentage of cTfr cells than advanced stage BC patients $(28.52 \% \pm 10.75 \%$ vs. $18.69 \% \pm 5.19 \%$; $\mathrm{t}=3.31$; $\mathrm{P}=0.006$; see Figure 1C). 

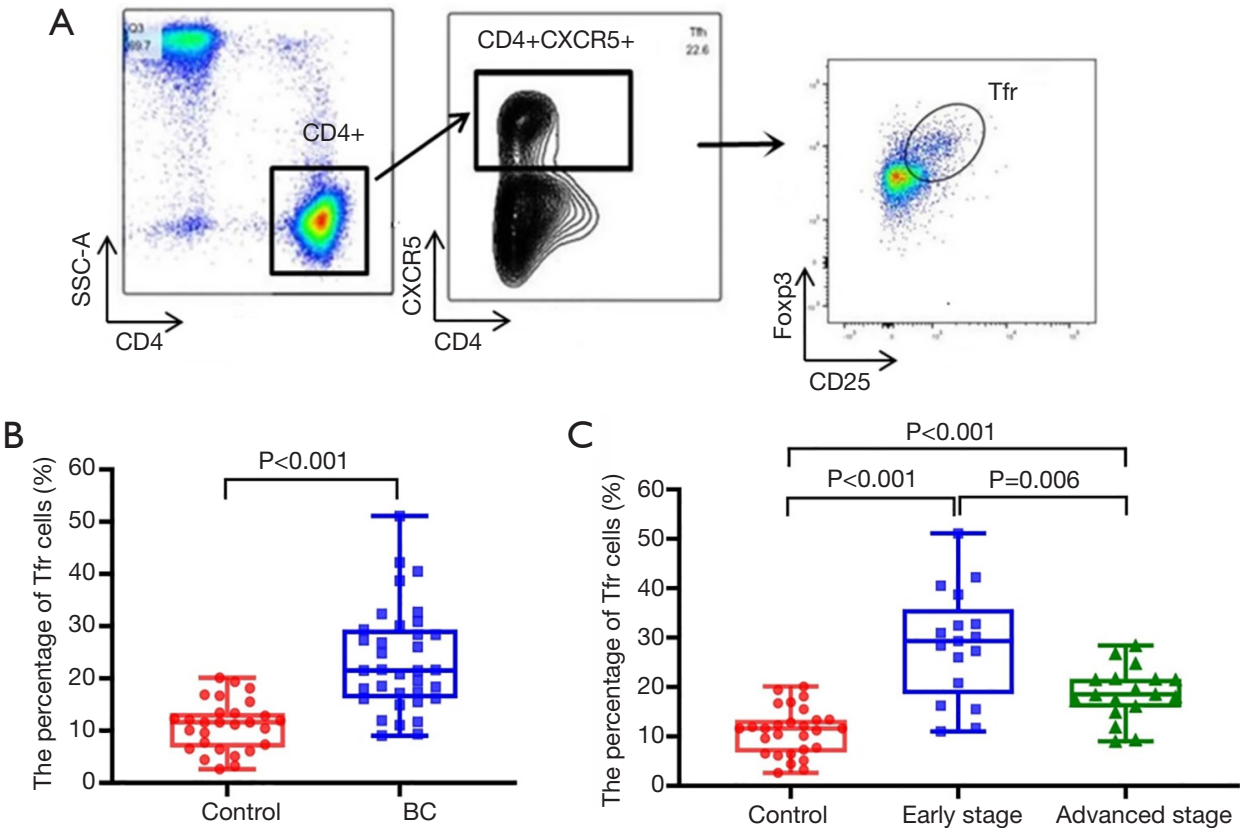

Figure 1 Circulating follicular regulatory $\mathrm{T}$ (c'Tfr) cells in breast cancer (BC) patients in different clinical stages and control patients. (A) FACS plots of CXCR5 and Foxp3 expression in CD4+ T cells from lymphocytes; (B) the percentage of cTfr cells from CD4+ T cells in BC patients and healthy control patients; (C) the percentage of cTfr cells from CD4+ T cells in early stage disease BC patients, advanced stage disease BC patients, and healthy control patients.

\section{The relationship of $c$ Tfr cells with different histological types}

We next examined the relationships between cTfr cells and molecular types in BC patients. As Figure $2 A$ shows, there was no significant difference in cTfr cells between the hormone receptor positive (HR+) BC patients and hormone receptor negative (HR-) patients $(26.07 \% \pm 10.07 \%$ and $20.66 \% \pm 8.72 \%$, respectively; $\mathrm{P}=0.141$ ). However, the percentages of cTfr cells in TNBC patients were significantly higher than those of HER2+and luminal BC patients $(28.25 \% \pm 10.11 \%$ vs. $22.32 \% \pm 8.82 \% ; \mathrm{P}=0.128$; $28.25 \% \pm 10.11 \%$ vs. $18.50 \% \pm 8.15 \% ; \mathrm{P}=0.028$ ); however, there was no significant difference between the HER2+ and luminal BC patients $(22.32 \% \pm 8.82 \%$ vs. $18.5 \% \pm 8.15 \%$; $\mathrm{t}=0.473 ; \mathrm{P}=0.432$; see Figure $2 B$ ).

\section{CXCR5+Foxp3+Tfr cells in the peripheral blood of early- stage $B C$ patients were negatively correlated with $L D H$}

To investigate the significance of the increased percentage of cTfr cells in BC patients, we analyzed the correlation between the percentage of cTfr cells and serum LDH levels in BC patients. The results showed that the serum $\mathrm{LDH}$ levels of early-stage BC patients were significantly lower than those of advanced stage BC patients $(271.53 \pm 48.87$ vs. $257.45 \pm 13.80 ; \mathrm{P}=0.036$ ). The percentage of circulating CXCR5+Foxp3+Tfr cells was significantly negatively correlated with serum LDH levels in early-stage patients $(\mathrm{r}=-0.585 ; \mathrm{P}=0.008$; see Figure $3 A)$, but was not significantly correlated with serum LDH levels in advanced stage $\mathrm{BC}$ patients $(\mathrm{P}=0.875$; see Figure $3 B)$.

\section{The increased expression of TIM-3 on the surface of Tfr cells in TNBC patients}

To explore the significance of increased Tfr cells and the specific pathway of action in BC patients, we analyzed the expression of TIM-3 on the surface of cTfr cells in the peripheral blood of TNBC patients and healthy control patients using mass spectrometry. As Figure $4 A-4 B$ show, we analysed the expression of TIM-3 and Foxp3 of cTfr cells in TNBC and healthy control patients, the results revealed that the frequency of TIM-3 of BC patients was significantly higher than that of healthy patients $(3.93 \pm 0.92$ vs. $2.65 \pm 0.15$, respectively; $\mathrm{t}=-3.02 ; \mathrm{P}<0.05)$. However, there was no significant difference in the expression of TIM-3 between early-stage BC patients who had not undergone surgery 

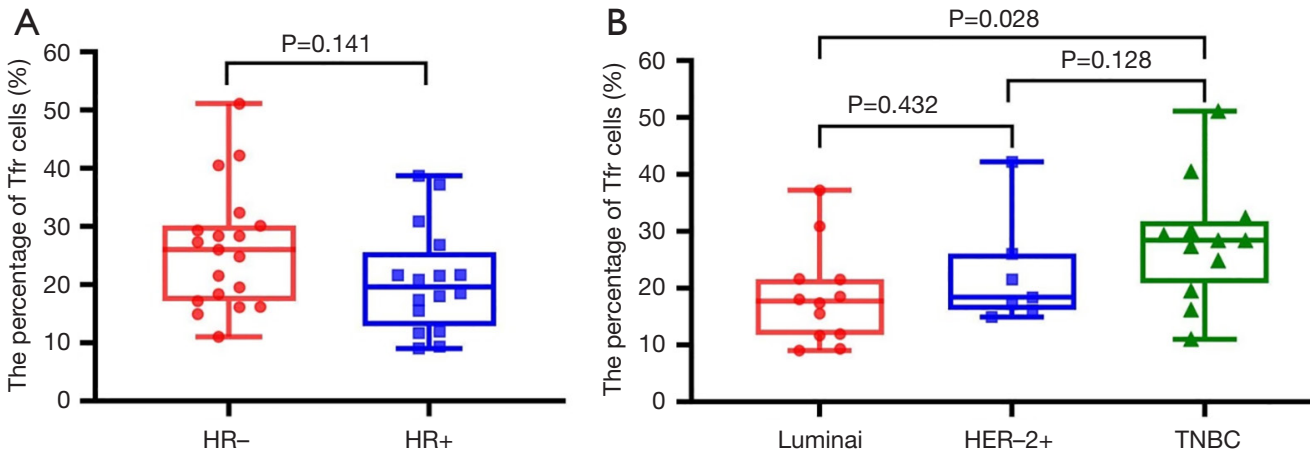

Figure 2 Circulating follicular regulatory $\mathrm{T}$ (c'Tfr) cells in breast cancer (BC) patients with histological subtype. (A) The percentage of c'Tfr cells from CD4+ T cells in BC patients with HR+ and HR-; (B) the percentage of c'Tfr cells from CD4+ T cells in triple-negative BC (TNBC), luminal BC, and HER2+ BC patients.
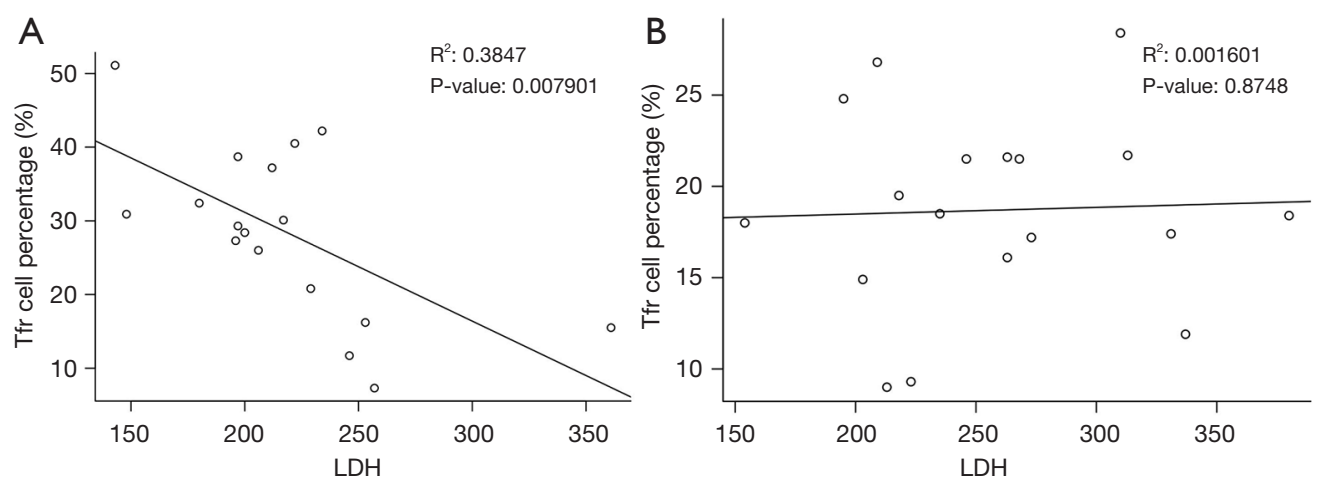

Figure 3 Correlation analysis between CXCR5+FoxP3+Tfr cells and lactate dehydrogenase (LDH) in breast cancer (BC) patients. (A) LDH levels in serum were negatively correlated with CXCR5+Foxp3+Tfr cells in early-stage BC patients; (B) the correlations were not significant between CXCR5+Foxp3+Tfr cells and LDH levels in the serum of advanced stage BC patients. Tfr, follicular regulatory T cells.

and advanced stage $\mathrm{BC}$ patients whose condition was evaluated as disease progression (PD). Next, we conducted a correlation analysis to examine the expression of TIM-3 and Foxp3 in TNBC patients. The results showed that there was a significant positive correlation between the expression of TIM-3 and Foxp3 in the cells ( $\mathrm{r}=0.82 ; \mathrm{P}=0.036$; see Figure $4 C$ ). Furthermore, the expression level of TIM-3 in different cell clusters were shown by a Spade tree diagram (Figure 4D) and UMAP scatter plot (Figure 4E).

\section{The expression of TIM-3 of Tfr cells in TNBC was negatively correlated with the IgG and CD19}

We also conducted a correlation analysis of the expression of TIM-3 in the Tfr cells and immunoglobulin ( $\mathrm{IgG}$ ) in the serum of TNBC patients. As Table 2 shows, the healthy control patients possessed higher levels of IgG than the TNBC patients $(0.78 \pm 1.0$ vs. $0.28 \pm 0.17 ; \mathrm{P}<0.05)$, and the expression of IgG was negatively correlated with TIM$3(\mathrm{r}=-0.98 ; \mathrm{P}<0.05)$. We then compared the expression of CD19 on the surface of B cells in the peripheral blood between TNBC and healthy control patients. Similar to IgG, the expression of CD19 of TNBC patients was significantly lower than that of healthy control patients $(0.12 \pm 0.10$ vs. $0.27 \pm 0.11 ; \mathrm{P}<0.05)$, and it was negatively correlated with TIM-3 expression on the surface of Tfr cells $(r=-0.98 ; \mathrm{P}<0.05)$. However, in relation to the analysis of $\mathrm{CD} 8$, no significant correlations were found.

\section{Discussion}

In this study, we found that the level of CXCR5+FoxP3+Tfr cells was increased in the peripheral blood of $\mathrm{BC}$ patients, especially in early-stage BC and TNBC patients. Further, we found a positive correlation between CXCR5+FoxP3+Tfr cells and LDH. Additionally, we 

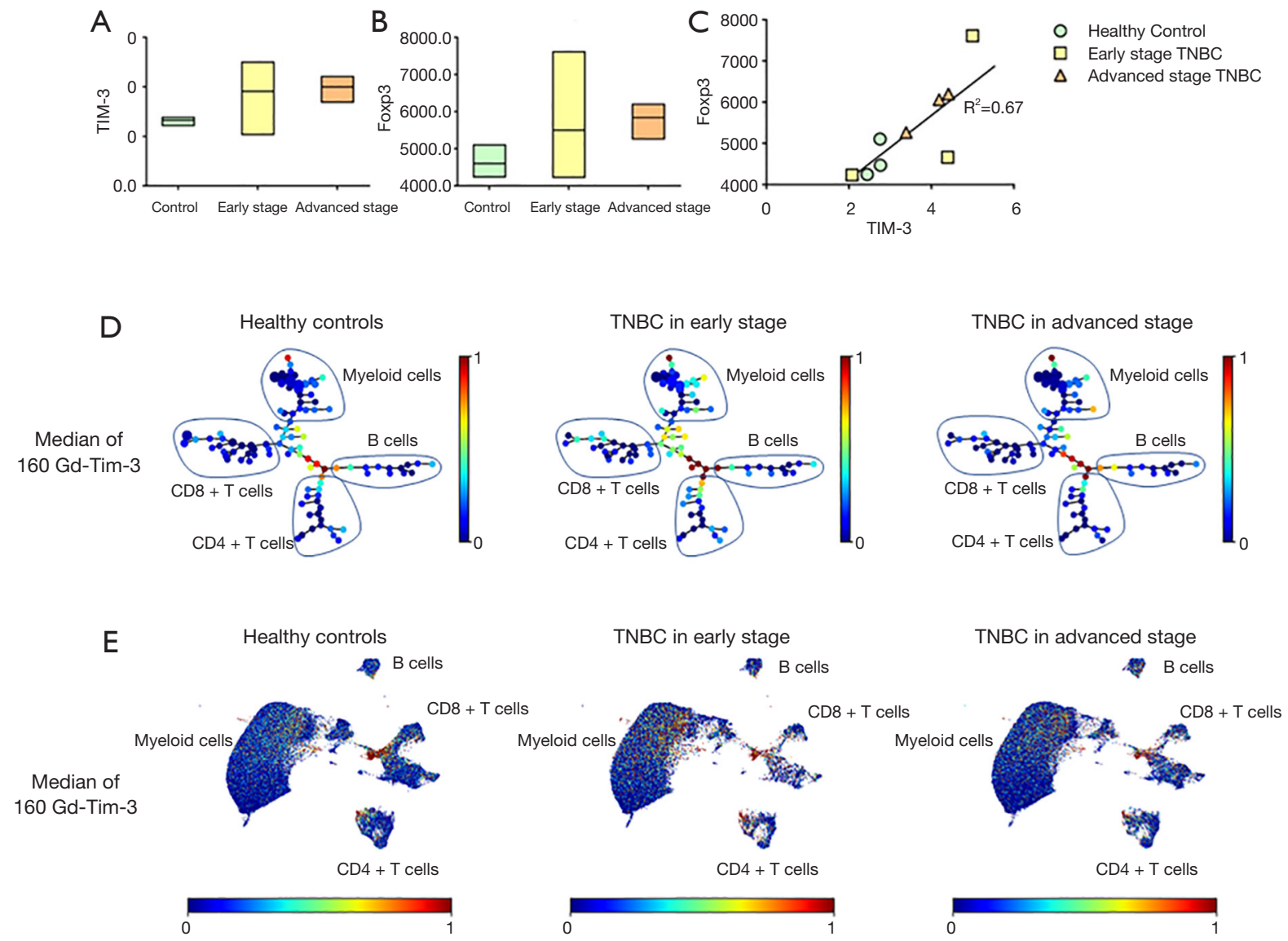

Figure 4 The expression of T-cell immunoglobulin mucin 3 (TIM-3) on the surface of circulating follicular regulatory T (cTfr) cells in triple-negative breast cancer (TNBC). (A) The expression of TIM-3 on the surface of cTfr cells in TNBC and healthy control patients; (B) the expression of Foxp3 of cTfr cells in TNBC and healthy control patients; (C) the correlation analysis between TIM-3 expression and Foxp3; (D,E) the expression level of TIM-3 in different cell clusters were shown by a Spade tree diagram (D) and UMAP scatter plot (E).

showed that the expression level of TIM-3 on the surface of Tfr cells was also significantly higher in TNBC patients than healthy control patients, and was negatively correlated with the related auto-antibodies. Our results provide significant insights into the role of $\mathrm{Tfr}$ cells in the pathogenesis process of $\mathrm{BC}$.

Studies have shown that Tfr cells play an important role in the development of autoimmune diseases and tumor diseases; for example, the frequency of FoxP3+CXCR5+CD4+Tfr cells in the peripheral blood of diffuse large $B$ cell lymphomas(DLBCL) patients was reported to be significantly increased compared to that of healthy control patients, especially in patients with less advanced stages of DLBCL, and the Tfr cells may be involved in intratumoral immunity (6). Additionally, the results of research on $\mathrm{Tfr}$ cells in NSCLC are similar to those for DLBCL. However, very few studies have been conducted on the function of
Tfr cells in BC. Indeed, to date, only Hongsheng Song et al. appeared to have examined the expression and characteristics of Tfr cells in BC (17). In the present study, we found an increase of cTfr cells in BC patients, and that the number of Tfr cells was significantly higher in early-stage BC patients than advanced stage. Thus, elevated cTfr cells might be a response to the pathogenesis of $\mathrm{BC}$, and might be related to the disease stage, such that patients with early-stage BC may have stronger immunosuppression than advanced stage $\mathrm{BC}$ patients.

We also retrospectively collected the serum $\mathrm{LDH}$ records of these patients, and found that LDH levels in early-stage $\mathrm{BC}$ patients were significantly lower than LDH levels in advanced stage BC patients. However, we also found that higher CXCR5+FoxP3+Tfr levels were correlated with lower levels of $\mathrm{LDH}$ in early-stage $\mathrm{BC}$ patients. $\mathrm{LDH}$ is an enzyme that catalyzes the interconversion of pyruvate and 
lactate, which in turn promotes cancer cell invasion, anoikis resistance, and tumor metastasis (19). Several studies have reported that LDH is a poor prognostic factor in BC $(20,21)$. Further, it has been suggested that LDH depletion inhibits the formation of metastases and prolongs the survival of mice with breast tumors by regulating the TME and modulating the immune response $(20,21)$. Tfr cells may be a better indicator of immune suppression in the germinal center of early BC patients than LDH. Notably, there were no significant correlations between CXCR5+FoxP3+cells and $\mathrm{LDH}$ levels in advanced stage $\mathrm{BC}$ patients; however, these results could be related to this study's small sample size and the different metastatic sites.

Compared to luminal BC, TNBC and HER2 + BC have higher tumor infiltrating lymphocytes (TILs) $(22,23)$. TIL infiltration is closely related to the prognosis of $\mathrm{BC}$ patients and their response after immunotherapy. Numerous studies have shown that the expression of immune checkpoints in tumor cells with high TIL infiltration may be higher that of tumor cells with low TIL infiltration $(14,24)$. In addition, according to a report of Saleh et al. (18), the coblockade of PD-1 and programmed death-ligand 1 (PD-L1) could further upregulate the co-expression of TIM- 3 and Lymphocyte activation gene-3 (LAG-3) on CD4+CD25+ $\mathrm{T}$ cells and CD4+CD25+Foxp3+Tregs in the presence of TNBC cells. Our results showed that CXCR5+FoxP3+Tfr cells were significantly more abundant in TNBC patients than HER2 + and luminal BC patients; however, no significant difference was found between TNBC and HER2 + BC patients.

Most importantly, we showed that the expression of TIM-3 on the surface of Tfr cells was significantly higher than that of healthy control patients. We also observed a positive relationship between the expression of TIM-3 and Foxp3, which distinguishes Tfr cells from Tfh cells, and a negative correlation between the expression of TIM-3 and IgG on the surface of B lymphocytes. Thus, we speculate that the elevated expression of TIM-3 on the surface of Tfr cells might mediate tumor immune suppression in BC patients through the surface TIM-3, and affect the proliferation of effector cells in the germinal center, thus inhibiting the occurrence of humoral immunity.

Overall, our study showed an increased percentage of CXCR5+FoxP3+Tfr cells in BC patients. We theorize that this might play an important function in the pathogenesis of BC. Our research on the pathway of action of Tfr cells in 6 TNBC patients was only preliminary; thus, the potential mechanisms require further research.

\section{Acknowledgments}

Funding: This work was supported by the National Natural Science Foundation of China (No. 81672597).

\section{Footnote}

Reporting Checklist: The authors have completed the MDAR reporting checklist. Available at https://dx.doi. org/10.21037/atm-21-3848

Data Sharing Statement: Available at https://dx.doi. org/10.21037/atm-21-3848

Conflicts of Interest: All authors have completed the ICMJE uniform disclosure form (available at https://dx.doi. org/10.21037/atm-21-3848). The authors have no conflicts of interest to declare.

Ethical Statement: The authors are accountable for all aspects of the work in ensuring that questions related to the accuracy or integrity of any part of the work are appropriately investigated and resolved. All procedures performed in this study involving human participants were in accordance with the Declaration of Helsinki (as revised in 2013). The study was approved by institutional ethics board of Cancer Hospital of the University of Chinese Academy of Sciences/Zhejiang Cancer Hospital (No.: IRB2020-14). Individual consent for this retrospective analysis was waived.

Open Access Statement: This is an Open Access article distributed in accordance with the Creative Commons Attribution-NonCommercial-NoDerivs 4.0 International License (CC BY-NC-ND 4.0), which permits the noncommercial replication and distribution of the article with the strict proviso that no changes or edits are made and the original work is properly cited (including links to both the formal publication through the relevant DOI and the license). See: https://creativecommons.org/licenses/by-nc-nd/4.0/.

\section{References}

1. Vanderleyden I, Fra-Bido SC, Innocentin S, et al. Follicular Regulatory T Cells Can Access the Germinal Center Independently of CXCR5. Cell Rep 2020;30:611-619.e4.

2. Clement RL, Daccache J, Mohammed MT, et al. Follicular regulatory $\mathrm{T}$ cells control humoral and allergic immunity by restraining early B cell responses. Nat Immunol 
2019;20:1360-71.

3. Hou S, Clement RL, Diallo A, et al. FoxP3 and Ezh2 regulate $\mathrm{Tfr}$ cell suppressive function and transcriptional program. J Exp Med 2019;216:605-20.

4. Guo Z, Liang $\mathrm{H}, \mathrm{Xu} \mathrm{Y}$, et al. The role of circulating $\mathrm{t}$ follicular helper cells and regulatory cells in non-small cell lung cancer patients. Scand J Immunol 2017;86:107-12 .

5. Rao DA, Gurish MF, Marshall JL, et al. Pathologically expanded peripheral T helper cell subset drives B cells in rheumatoid arthritis. Nature 2017;542:110-4.

6. Nedelkovska H, Rosenberg AF, Hilchey SP, et al. Follicular Lymphoma Tregs Have a Distinct Transcription Profile Impacting Their Migration and Retention in the Malignant Lymph Node. PLoS One 2016;11:e0155347.

7. Cha Z, Gu H, Zang Y, et al. The prevalence and function of CD4+CXCR5+Foxp3+ follicular regulatory $\mathrm{T}$ cells in diffuse large B cell lymphoma. Int Immunopharmacol 2018;61:132-9.

8. Brummelman J, Mazza EMC, Alvisi G, et al. Highdimensional single cell analysis identifies stem-like cytotoxic CD8+ T cells infiltrating human tumors. J Exp Med 2018;215:2520-35.

9. Yang X, Jiang X, Chen G, et al. T cell Ig mucin-3 promotes homeostasis of sepsis by negatively regulating the TLR response. J Immunol 2013;190:2068-79.

10. Siddiqui I, Schaeuble K, Chennupati V, et al. Intratumoral Tcf1+PD-1+CD8+ T Cells with Stem-like Properties Promote Tumor Control in Response to Vaccination and Checkpoint Blockade Immunotherapy. Immunity 2019;50:195-211.e10.

11. Wang X, Qi Y, Kong X, et al. Immunological therapy: A novel thriving area for triple-negative breast cancer treatment. Cancer Lett 2019;442:409-28.

12. Keren L, Bosse M, Marquez D, et al. A Structured TumorImmune Microenvironment in Triple Negative Breast Cancer Revealed by Multiplexed Ion Beam Imaging. Cell 2018;174:1373-1387.e19.

13. Schmid P, Adams S, Rugo HS, et al. Atezolizumab and Nab-Paclitaxel in Advanced Triple-Negative Breast Cancer. N Engl J Med 2018;379:2108-21.

14. Denkert C, Loibl S, Noske A, et al. Tumor-associated lymphocytes as an independent predictor of response to neoadjuvant chemotherapy in breast cancer. J Clin Oncol 2010;28:105-13. Erratum in: J Clin Oncol 2010;28:708.

15. Zheng J, Wang T, Zhang L, et al. Dysregulation of Circulating Tfr/Tfh Ratio in Primary biliary cholangitis. Scand J Immunol 2017;86:452-61.
16. Wagner J, Rapsomaniki MA, Chevrier S, et al. A SingleCell Atlas of the Tumor and Immune Ecosystem of Human Breast Cancer. Cell 2019;177:1330-45.e18.

17. Song H, Liu A, Liu G, et al. T follicular regulatory cells suppress Tfh-mediated B cell help and synergistically increase IL-10-producing B cells in breast carcinoma. Immunol Res 2019;67:416-23.

18. Saleh R, Toor SM, Khalaf S, et al. Breast Cancer Cells and PD-1/PD-L1 Blockade Upregulate the Expression of PD1, CTLA-4, TIM-3 and LAG-3 Immune Checkpoints in CD4+ T Cells. Vaccines (Basel) 2019;7:149.

19. Serganova I, Cohen IJ, Vemuri K, et al. LDH-A regulates the tumor microenvironment via HIF-signaling and modulates the immune response. PLoS One 2018;13:e0203965.

20. Chen B, Dai D, Tang H, et al. Pre-treatment serum alkaline phosphatase and lactate dehydrogenase as prognostic factors in triple negative breast cancer. J Cancer 2016;7:2309-16.

21. Jin L, Chun J, Pan C, et al. Phosphorylation-mediated activation of LDHA promotes cancer cell invasion and tumour metastasis. Oncogene 2017;36:3797-806.

22. Loi S, Sirtaine N, Piette F, et al. Prognostic and predictive value of tumor-infiltrating lymphocytes in a phase III randomized adjuvant breast cancer trial in node-positive breast cancer comparing the addition of docetaxel to doxorubicin with doxorubicin-based chemotherapy: BIG 02-98. J Clin Oncol 2013;31:860-7.

23. Loi S, Michiels S, Salgado R, et al. Tumor infiltrating lymphocytes are prognostic in triple negative breast cancer and predictive for trastuzumab benefit in early breast cancer: results from the FinHER trial. Ann Oncol 2014;25:1544-50.

24. Salgado R, Denkert C, Campbell C, et al. TumorInfiltrating Lymphocytes and Associations With Pathological Complete Response and Event-Free Survival in HER2-Positive Early-Stage Breast Cancer Treated With Lapatinib and Trastuzumab: A Secondary Analysis of the NeoALTTO Trial. JAMA Oncol 2015;1:448-54.

(English Language Editor: L. Huleatt)

Cite this article as: Miao X, Guo Q, Pan Z, Xu X, Shao X, Wang $X$. The characteristics and novel clinical implications of CD4+CXCR5+Foxp3+ follicular regulatory $\mathrm{T}$ cells in breast cancer. Ann Transl Med 2021;9(16):1332. doi: 10.21037/atm21-3848 